\title{
Evaluación de la viabilidad de huevos de abeja Apis mellifera L. sometidos a radiación ultravioleta
}

\author{
Evaluation of the viability of bee eggs Apis mellifera L. when submited \\ to ultra-violet radiation
}

\author{
Ximena Araneda D. ${ }^{1}$; Elizabeth Grandón S. ${ }^{1}$; Paul Escobar B. ${ }^{1}$; José Luis Barahona M. ${ }^{1}$
}

\begin{abstract}
RESUMEN
En esta investigación se evaluó la viabilidad de huevos de Apis mellifera L. expuestos a radiación UV-A y UV-B mediante un tubo fluorescente con espectro entre los $280 \mathrm{~nm}$ a los $720 \mathrm{~nm}$ de 30 watts de potencia. El diseño experimental fue completamente al azar evaluándose viabilidad con tiempos de exposición de $0 \mathrm{~s}, 10 \mathrm{~s}, 30 \mathrm{~s}, 60 \mathrm{~s}$ y $90 \mathrm{~s}$. El método de evaluación fue la presencia de celdillas operculadas al noveno día después de exponer los huevos de 24 horas a radiación. Los resultados obtenidos de las medias entre los distintos tiempos de exposición demostraron que existen diferencias estadísticamente significativas entre los tratamientos. Se presentó una mayor viabilidad a $0 \mathrm{~s}$ con $89,26 \%$, para $10 \mathrm{~s}$ de exposición 56,59\%, para 30 s 36,47\%, para 60 s $31,46 \%$ y para $90 \mathrm{~s}$ de exposición $21,83 \%$ de viabilidad. Con este experimento se obtienen valiosos antecedentes sobre los cuales trabajar a fin de reducir pérdidas en crías de abejas, las cuales son sensibles a exposiciones de radiación UV.
\end{abstract}

Palabras clave: Apis mellifera L., espectro de radiación, radiación UV, viabilidad.

\begin{abstract}
This study evaluated the viability of the eggs of Apis mellifera $L$. (honeybee) when exposed to UV-A and UV-B radiation using a fluorescent tube of 30 watts power with a spectrum between $280 \mathrm{~nm}$ and $720 \mathrm{~nm}$. The design of the experiment was completely random, with evaluations of viability after exposure times of $0,10,30,60$ and 90 seconds. The evaluation method was the presence of operculate cells on the ninth day after exposure of 24-hour old eggs to radiation. The results obtained from the measurements among the different exposure times showed that there were statistically significant differences between the treatments. The greatest viability occurred at 0 exposure with $89.26 \%$, followed by $56.59 \%$ for $10 \mathrm{~s}$ exposure, $36.47 \%$ for $30 \mathrm{~s}, 31.46 \%$ for $60 \mathrm{~s}$, and $21.83 \%$ viability with 90 s exposure. This experiment provides valuable information with which to reduce bee losses in breeding programmes, since they are sensitive to exposure to $U V$ radiation.
\end{abstract}

Key words: Apis mellifera L., radiation spectrum, UV radiation, viability.

\section{Introducción}

La luz natural comprende la región del espectro electromagnético que va desde el espectro ultravioleta invisible pasando por el espectro de luz visible hasta el infrarrojo invisible (Gliessman, 2002). La radiación ultravioleta (UV) se extiende dentro del espectro electromagnético, entre los $100 \mathrm{~nm}$ y 400 nm (Björn, 1999; Mc Kenzie et al., 2003). Se caracteriza por su alta frecuencia y alto nivel energético, siendo particularmente efectiva en inducir reacciones fotoquímicas (Hopkins, 1999) y se subdivide en tres bandas: UV-A (400 $\mathrm{nm}$ a $320 \mathrm{~nm}$ ), UV-B (320 nm a $280 \mathrm{~nm})$ y UV-C (280 nm a 100 nm) (Diffey, 1991). Siendo aquellos rayos UV con longitud de onda inferior a $200 \mathrm{~nm}$ potencialmente letales para los organismos vivos (Gliessman, 2002). No obstante, longitudes de onda menores que $290 \mathrm{~nm}$ es poco probable que alcancen la superficie terrestre, excepto en las altas altitudes (Diffey, 1991). Sin embargo, la contaminación industrial ha producido la disminución de la concentración de ozono estratosférico, lo que ha provocado un significativo aumento de la radiación ultravioleta-B

1 Universidad Católica de Temuco, Campus Norte, Rudecindo Ortega 02950, Temuco, Casilla 15-D. E-mail: xaraneda@ uct.cl. 
(UV-B) a nivel de la tropósfera (Frederick et al., 1989). En este sentido Posso (1999) indica que el ozono juega un papel crítico en el balance ecológico de la Tierra debido a su fuerte absorción de la luz UV altamente dañina. Por lo tanto, en la medida que el ozono estratosférico disminuya, los niveles de radiación UV-B se incrementarán (Björn, 1999). Tal como ocurre en la zona Austral de América del Sur (Cabrera et al., 1995; Rivas et al., 2002), causando efectos adversos en cada uno de los organismos en el medio ambiente, y los procesos de los ecosistemas (Solomon, 2008), tanto sobre el medio ambiente terrestre y acuático (Day y Neale, 2002), provocando en este último alteraciones en la calidad nutricional de los alimentos (fitoplancton marino) disponibles para los niveles tróficos superiores (Skerratt et al., 1998).

Entre los daños a los seres humanos provocados por la radiación UV-B, se encuentran la incidencia de cataratas de los ojos y el cáncer de la piel, como melanoma maligno (Solomon, 2008), y daños como el eritema (Lovengreen et al., 2002). En las plantas se han observado cambios fisiológicos, bioquímicos, morfológicos y anatómicos, afectando el crecimiento vegetal (Diffey, 1991), como por ejemplo en avena (Ruhland et al., 2007). Pero también se observan efectos significativos sobre la herbivoría por insectos (Ballare et al., 1996; Caputo et al., 2006), afectando además, al próximo nivel trófico (depredadores) (Foggo et al., 2007). Igualmente, se han observado efectos sobre la corteza del suelo, disminuyendo la productividad y aumentando la mortalidad biológica de los suelos (Belnap et al., 2007). En organismos acuáticos la exposición prolongada a la radiación UV-B ha provocado daños (Mazza et al., 1999), como aumento de la mortalidad embrionaria en especies de anfibios (Anzalone et al., 1998), mortalidad significante en huevos de bacalao atlántico (Béland et al., 1999), daños y mortalidad en invertebrados de agua dulce (Hurtubise et al., 1998; Cywinska et al., 2000). Los efectos de la radiación UV también se han observado en insectos, como por ejemplo fotosensibilidad a la radiación UV en Caliothrips phaseoli (Mazza et al., 2002) y el género Deleatidium (Johansson y Nyström, 2004), reactivación biológica y desaparición de alrededor de $7 \times 10^{9}$ dímeros de pirimidina del ARN total por huevos de Smittia sp. (Kalthoff et al., 1971), reducción en el peso de larvas, crisálidas y adultos en gusanos de seda, disminución en el peso del capullo y mortalidad en las larvas al aumentar la dosis de radiación UV (Faruki y Kundu, 2005), disminución en la ovoposición de Callosobruchus chinensis L. (Sharma y Dwivedi, 1997), inactivación en huevos de Sciara ocellaris con radiaciones UV de $254 \mathrm{~nm}$ o $300 \mathrm{~nm}$ de longitud de onda (Perondini, 1984), disminución gradual en el porcentaje de eclosión de los huevos de Tribolium castaneum, T. cofusum y Cadra cautella con radiación UV de longitud de onda de $254 \mathrm{~nm}$ con diferentes duraciones (Faruki et al., 2007), actividad mutagénica a nivel de las alas al exponer larvas de Drosophila melanogaster a diferentes longitudes de onda de rayos UV-A (Negishi et al., 2001), deformaciones en diferentes etapas de desarrollo y aumentos de mortalidad por efecto de la radiación UV en Alphitobius diaperinus (Faruki et al., 2005). En huevos de Apis mellifera L., con 10 segundos de exposición al sol directo en el Sur de Chile o Brasil, la mortalidad es mayor del 50\% (Araneda, 1997), también se observan efectos en la viabilidad, retrasos del desarrollo y aumentos en la mortalidad de larvas de abejas en condiciones naturales de radiación solar (González, 2007).

En este contexto resulta importante tener mayor información de los efectos de la luz UV sobre las abejas A. mellifera considerando que sus estados inmaduros son sensibles y analizando la importancia que tienen estos insectos en la polinización de diversos cultivos de importancia económica. En este trabajo se pretende evaluar el efecto de la exposición a radiación UV-A y UV-B en base a la viabilidad de los huevos de abeja obrera bajo condiciones experimentales.

\section{Materiales y Métodos}

La investigación se llevó a cabo en el Centro Experimental Pillanlelbún, perteneciente a la Universidad Católica de Temuco, ubicado a 18 kilómetros al Norte de la ciudad de Temuco $\left(38^{\circ}\right.$ 39' 17,2" lat. Sur, $72^{\circ} 26^{\prime}$ 56" long. Oeste), en la comuna de Lautaro, provincia de Cautín, Región de La Araucanía, Chile.

Se utilizaron cinco núcleos de cinco marcos cada uno, dos colmenas tipo Langstroth con alza y rejilla excluidora de reina.

Para comparar viabilidad en huevos de abejas obreras expuestas a radiación UV-A y UV-B, se utilizaron distintos tiempos de exposición siendo éstos T0: $0 \mathrm{~s}, \mathrm{~T} 1: 10 \mathrm{~s}, \mathrm{~T} 2: 30 \mathrm{~s}, \mathrm{~T} 3: 60 \mathrm{~s}$ y T4: $90 \mathrm{~s}$. Para ello se construyó una caja de madera de $4 \mathrm{~mm}$ de espesor, cuyas medidas fueron 1 metro de largo, 
por $50 \mathrm{~cm}$ de alto y $50 \mathrm{~cm}$ de fondo. Dentro de esta caja se instaló un tubo fluorescente modelo repti glo 5,0 de 30 watts a $30 \mathrm{~cm}$ de distancia e intensidad UV-A y UV-B de $1,02 \mathrm{~W} \mathrm{~m}^{-2}$.

Para la obtención de huevos se introdujo un marco limpio al núcleo. Después de 24 horas se revisó el marco para ver postura efectiva, se llevó a laboratorio y se procedió a contar las celdillas con huevos, luego se irradió cada marco dentro de la caja a $30 \mathrm{~cm}$ del tubo según el tratamiento a realizar.

Luego de realizados los tratamientos, se llevaron los marcos a incubar a la segunda alza de una colonia vigorosa, con rejilla excluidora para evitar nuevas posturas de la reina en caso de haber mortalidad de los huevos, transcurridos 9 días desde el momento de la postura se verificó la presencia de celdillas operculadas, lo que indicó viabilidad de la cría.

Se medió la irradiancia espectral $\left(\mathrm{W} \mathrm{m}^{-2} \mathrm{~nm}\right)$ del tubo fluorescente con un espectro radiómetro, correspondiente a la longitud de onda de la luz UV-B desde los $280 \mathrm{~nm}$ a los $315 \mathrm{~nm}$ y UV-A que va desde los $315 \mathrm{~nm}$ a los $400 \mathrm{~nm}$, estos valores fueron comparados con la irradiancia espectral proveniente del sol en un día despejado del mes de febrero a las 14:00 h, horario en el cual los rayos ultravioleta alcanzan de forma más directa la superficie de la tierra. Luego se procedió a medir la irradiancia integral del tubo fluorescente y la irradiancia integral del sol. La irradiancia integral se obtiene de la sumatoria de la espectral, siendo el resultado la sumatoria desde los $280 \mathrm{~nm}$ a los $400 \mathrm{~nm}$, espectro en el cual están contenidas la radiación UV-A y UV-B, siendo para el sol 53,31 $\mathrm{W} \mathrm{m}{ }^{-2}$ y para el tubo $1,02 \mathrm{~W} \mathrm{~m}^{-2}$.

Se realizó un ANDEVA para el diseño experimental de una vía completamente al azar $(\mathrm{P} \leq 0,05)$. Se utilizó la prueba de Levene para verificar que los datos cumplieran los supuestos estadísticos de distribución normal y homogeneidad de varianzas y la prueba de comparación múltiple de Duncan, para grupos homogéneos. Los datos fueron analizados en el programa estadístico SPSS 12.0.

\section{Resultados y Discusión}

El análisis de los datos arrojó diferencias significativas en el promedio de viabilidad entre los tratamientos (Tabla 1).

A partir de los resultados de la prueba de comparación múltiple de promedios de Duncan, se puede
Tabla 1. Porcentaje de viabilidad promedio para los distintos tratamientos

\begin{tabular}{lcc}
\hline Tratamientos & Cría viva $(\%)$ & Desviación estándar \\
\hline T0 $(0 \mathrm{~s})$ & $89,26 \mathrm{a}$ & $\pm 3,8621$ \\
T1 $(10 \mathrm{~s})$ & $56,59 \mathrm{~b}$ & $\pm 8,9539$ \\
T2 $(30 \mathrm{~s})$ & $36,47 \mathrm{c}$ & $\pm 24,4148$ \\
T3 $(60 \mathrm{~s})$ & $31,46 \mathrm{c}$ & $\pm 18,3564$ \\
T4 $(90 \mathrm{~s})$ & $21,83 \mathrm{c}$ & $\pm 9,5331$ \\
\hline
\end{tabular}

Letras distintas señalan diferencias significativas según test de Duncan $(\mathrm{P} \leq 0,05)$.

concluir que, entre los grupos comparados el que corresponde al tratamiento testigo con 0 segundos de exposición a luz UV es el que presentó mayor viabilidad ya que tiene el promedio más alto y con diferencias estadísticamente significativas respecto al resto, el segundo mayor promedio lo presentó el T1 con 10 segundos de exposición a luz UV presentando también diferencias estadísticas. Los tratamientos $\mathrm{T} 2, \mathrm{~T} 3$ y T4 quienes recibieron mayores exposiciones a luz UV $30 \mathrm{~s}, 60 \mathrm{~s}$, y $90 \mathrm{~s}$ respectivamente, comparten diferencias estadísticamente significativas con respecto a T0 y T1.

En estudios realizados por Araneda (1997), sobre abejas africanizadas de Brasil y europeas de Chile, encontró diferencias significativas en la viabilidad de huevos, ya que con exposiciones directas al sol encontró una viabilidad de $69,27 \%$ en abejas africanizadas y $39,58 \%$ en abejas europeas, ambas especies expuestas a una radiación de 2,44 $\mathrm{Med} \mathrm{h}^{-1}$, en cambio en condiciones artificiales huevos expuestos a una radiación de $0,36 \mathrm{Med} \mathrm{h}^{-1}$, mediante una lámpara UV, la viabilidad en abejas africanizadas fue de 4,4\% y de europeas es de 7,25\%. Entre un experimento y otro hay una diferencia de $49,34 \%$, esto se debe a que hubo cinco segundos de diferencia, además, la luz utilizada no fue la misma. Araneda (1997) utilizó una luz que no tiene espectro más allá de los $400 \mathrm{~nm}$, por lo tanto, no hay posibilidad de reparación al daño producido por la luz UV, en cambio, en este estudio la luz utilizada sí tiene espectro mayor a los $400 \mathrm{~nm}$, teniendo la posibilidad de reparar el daño producido aumentando la viabilidad; sin embargo, el grado de fotorreactivación disminuye con el aumento de dosis de UV (Quek et al., 2006), ya que Jäckle y Kalthoff (1977) reportaron que dosis de irradiación UV entre los $380 \mathrm{~nm}$ y $400 \mathrm{~nm}$ causa fotorreactivación en huevos de Smittia y monomerización de los dímeros 
de pirimidina en su ARN, así como también daños en distintas biomoléculas, entre las cuales la más importante es el ADN (Carrasco-Ríos, 2009).

En este estudio se puede observar que entre los menores porcentajes de viabilidad, la exposición a 90 s reportó un $21,83 \%$ de viabilidad de cría, distinto a lo reportado por González (2007), quien al evaluar la viabilidad sobre larvas de A. miellifera, expuestas a radiación directa del sol en las ciudades de Valdivia y Concepción con tiempos de exposición de tres minutos y distintos horarios, obtuvo a las 13:00 h un 27,6\% de viabilidad (febrero a marzo) en Concepción y un 34,2\% (enero) en Valdivia. Mientras que Araneda (1997), reportó a la misma hora un $32,33 \%$ de viabilidad y con $10 \mathrm{~s}$ de exposición. Lo que demuestra que dependiendo la hora de exposición a la radiación UV los porcentajes de viabilidad varían, incluso en zonas áridas como en el desierto de Atacama donde la viabilidad de los microorganismos a las 14:00 h se reduce drásticamente (Cockell et al., 2008), alcanzando los máximos valores UV cercano a las 13:29 h (Piacentini et al., 2003). Sin embargo, los incrementos altitudinales de la radiación UV son menores en áreas rurales (4\% $10 \%$ por kilometro) a lo largo de la costa y valles de los Andes, alcanzando magnitudes muy bajas ( $2 \%$ o menos) en las cumbres andinas de las regiones desérticas (Cabrera et al., 1995). No obstante, se han reportado valores extremos del índice ultravioleta en zonas del Altiplano (Arica y Zapahuira) en las estaciones del año correspondientes a primavera y verano para días de cielo claro y en horas cercanas al mediodía solar, aun en los meses de invierno, ya que durante estos meses se obtienen valores del índice solar ultravioleta considerados altos o extremos (Rivas et al., 2008).

En el presente experimento el grupo control presentó un porcentaje de viabilidad de $89,26 \%$, lo que indica un grado importante de mortalidad. El estudio realizado por González (2007) presentó un porcentaje de viabilidad para los grupos control de $81,4 \%$ y $72,5 \%$ en los meses de enero y febrero respectivamente en la ciudad de Valdivia y de 66,6\% entre febrero a marzo en Concepción, ambos en el año 2006, lo que indica que también hay un porcentaje de mortalidad. Esto se podría justificar por la capacidad que tienen las abejas de marcar los individuos con feromonas (olor característico de cada colonia), ya que al cambiar los panales de colmena al momento de incubar los individuos después de ser irradiados, las nodrizas de la colonia en la cual se incuban no las alimentan, y mueren por diferenciación de la cría, esto concuerda con lo que indica Martin et al. (2004), quienes manifiestan que abejas nodrizas diferencian huevos puestos por obreras y huevos puestos por reinas sólo por las feromonas, diferenciando reinas y crías de otras colonias sólo por el olor, siendo una señal muy sólida (Martin et al., 2005). Además podrían afectar la mortalidad de la cría factores externos no evaluados como humedad, temperatura, intensidad luminosa.

En el presente estudio, a través de la regresión lineal, el valor del coeficiente de determinación $\left(R^{2}=\right.$ 0,9076) demuestra que las variables en cuestión tienen una relación inversamente proporcional entre los cinco tratamientos, esto es, a medida que aumentan los tiempos de radiación de luz UV-A y UV-B los valores de viabilidad disminuyen. Similar a lo reportado por Araneda (1997) y González (2007), quienes al realizar análisis de correlación entre las variables de viabilidad y radiación UV-B en huevos de abeja y en la etapa larval de las abejas obreras (A. mellifera), en Brasil y Chile, respectivamente, también obtienen una correlación inversamente proporcional $\left(r=-0,65\right.$ en Brasil y $R^{2}$ de 0,9028 en Chile), quedando de manifiesto que al aumentar la radiación UV-B disminuye la viabilidad. Estos resultados se ajustan a los presentados por Machado (1986), que también llega a la misma conclusión, ya que estudiando el efecto de la radiación UV en abejas africanizadas verificó una disminución de la viabilidad, a medida que aumenta el tiempo de radiación. Machado (1991) concluyó en su trabajo que al exponer larvas de $0 \mathrm{~h}$ a $6 \mathrm{~h}$ de edad durante diferentes periodos de tiempo, observó que las viabilidades disminuían a medida que se aumentaba el tiempo de exposición, siendo esta viabilidad siempre menor que los grupos control. Tal como lo señalan Faruki y Kundu (2005), quienes registraron aumentos de mortalidad en larvas de Bómbix mori L. (Lepidóptera: Bombycidae) y en todos los estadíos de desarrollo debido a la radiación UV, ya que ésta afecta gravemente la progenie, incrementándose con el tiempo de exposición, tal como lo observado en $A$. diaperinus (Panzer) (Coleóptera: Tenebrionidae) por Begum et al. (2007). Lo que demuestra que la radiación UV puede ser un factor limitante importante en la viabilidad de los seres vivos como de los microorganismos, incluso hasta en regiones áridas como el desierto de Atacama, como lo reportado por Cockell et al. (2008), quienes investigaron daños en la viabilidad 
microbiana, demostrando que un día de exposición a la radiación UV es suficiente para causar la muerte celular de los organismos, principalmente en meses de verano donde se incrementa la radiación solar UV debido a la radiación difundida por el reflejo de las superficies (arena y mar) que rodean estas zonas, como por ejemplo Arica, zona muy pobre en vegetación (Rojas y Rivas, 2002).

En esta investigación los resultados obtenidos fueron de una fuente de radiación artificial y muestran una muy baja viabilidad, comparando el grupo control a 0 segundos y el último tratamiento a 90 segundos de exposición a luz UV, presentando un $89,26 \%$ y $21,83 \%$ respectivamente, ostentando una diferencia de $53,43 \%$ de viabilidad entre los dos tratamientos comparados. Los resultados concuerdan con aquellos presentados por Machado (1986; 1991), donde la viabilidad de huevos de obreras fue afectada por la radiación proveniente de una fuente artificial. Aquí las viabilidades disminuían a medida que aumentaba el tiempo de exposición. Mazza et al. (1999), en experimentos realizados en insectos detectaron una sensibilidad de éstos a la radiación UV-B, ya que los insectos evitan la exposición solar UV-B.

Según Faruki et al. (2007), comprobaron que la radiación UV reduce gradualmente la viabilidad de los huevos de T. castaneum, T. confusum y $C$. cautella en diferentes edades, siendo más sensibles mientras más jóvenes sean éstos.

Sin embargo, no sólo la viabilidad se ve afectada por la radiación UV, ya que Begum et al. (2007) reportaron que al exponer larvas de A. diaperinus (Panzer) con diferentes tiempos de exposición de radiación UV, ésta reduce significativamente la producción de huevos, afectándose progresivamente la fecundidad y fertilidad con el aumento de periodos de exposición. Del mismo modo González (2007) analizó individualmente las larvas de A. mellifera que sobrevivieron a las exposiciones de radiación solar para determinar el estado de desarrollo en el que se encontraban, obteniendo un retraso en el desarrollo normal de las abejas tanto en Valdivia como en Concepción, además reportó que la radiación solar afectó la coloración de los ojos, persistiendo el color púrpura y no el ceniza que es característico de las abejas adultas. Además, encontró un 5\% de abejas en estado adulto en posición invertida, atribuyendo el hecho a un efecto indirecto de la radiación UV-B sobre el alimento de las larvas, ya que al exponer los marcos con larvas a luz natural la radiación UV-B afecta la cantidad o concentración del alimento y el nivel nutricional de las larvas, disminuyendo el carácter inmuno-defensivo de las abejas manifestándose posiblemente un virus que determina la posición invertida.

Con los antecedentes recopilados se podría indicar a los apicultores el daño producido por exposiciones prolongadas y directas a la luz ultravioleta, y la consiguiente disminución de la población por este concepto, pudiendo generar algún tipo de protección a esta radiación.

\section{Conclusiones}

Se observó una fuerte disminución de la viabilidad de los huevos de A. mellifera expuestos a radiación ultravioleta UV-A y UV-B artificial, llegando a disminuir $67,43 \%$ al comparar el tratamiento testigo el cual no fue expuesto a radiación con el último tratamiento equivalente a 90 segundos el que presentó sólo una viabilidad de 21,83\%. Esto debido a que los estados inmaduros son extremadamente sensibles a este tipo de radiación, más aún cuando son exposiciones prolongadas y directas.

Existen diferencias significativas $(\mathrm{P} \leq 0,05)$ entre los cinco tratamientos estudiados, se demuestra que existen tres grupos. El primer grupo a $0 \mathrm{~s} \mathrm{de}$ exposición presenta un $89,26 \%$ de viabilidad, el segundo grupo a los $10 \mathrm{~s}$ presenta un $56,59 \%$ de viabilidad, y el tercer grupo a los $30 \mathrm{~s}, 60 \mathrm{~s}$ y $90 \mathrm{~s}$ comparten viabilidades similares siendo éstas $36,47 \% ; 31,46 \%$ y $21,83 \%$, respectivamente, no habiendo diferencias significativas en el último grupo. Esto indica claramente la disminución de la viabilidad de la cría provocados por exposiciones prolongadas y directas a luz UV; antecedentes que podrían ser de interés en futuras investigaciones con el fin de crear mecanismos para evitar pérdidas significativas por este concepto en apicultura.

Fue encontrada una correlación inversamente proporcional $\left(R^{2}=0,90\right)$ entre la viabilidad de huevos de abejas A. mellifera y tiempos de radiación UV entre los $0 \mathrm{~s}$ y $90 \mathrm{~s}$; a medida que aumentan los tiempos de exposición disminuye drásticamente la viabilidad de los huevos, se podría decir que desde $30 \mathrm{~s}$ de exposición hacia arriba hay una muy baja viabilidad cuando la cría es expuesta a radiación UV, lo que influiría considerablemente la prosperidad de la colonia. 


\section{Literatura Citada}

Anzalone, C.; Kats, L.; Gordons, M.

1998 Effects of solar UV-B radiation on embryonic development in Hyla cadaverina, Hyla regilla, and Taricha torosa. Conservation Biology. 12 (3): 646-653.

Araneda, X.

1997 Caracterizaçao da sensibilidade diferencial dos efeitos da luz UV em abelhas africanizadas e européias. Mestre em Ciéncias Área genética. Ribeirao Preto, Sao Paulo, Brasil. Universidade de Sao Paulo, Faculdade de Medicina de Ribeirao Preto. 87 p.

Ballare, C.L.; Scopel, A.L.; Stapleton, A.E.; Yanovsky, M.J. 1996 Solar ultraviolet-B radiation affects seedling emergence, DNA integrity, plant morphology, growth rate, and attractiveness to herbivore insects in Datura ferox. Plant Physiology. 112 (1): 161-170.

Begum, M.; Parween, S.; Faruki, S.

2007 Combined effect of UV-radiation and triflumuron on the progeny of Alphitobius diaperinus (Panzer) (Coleoptera: Tenebrionidae) at different storage period. Rajshahi University Zoological Society. 26: 4548.

Béland, F.; Browman, H.; Rodriguéz, C.; St-Pierre, J. 1999 Effects of solar ultraviolet radiation $(280-400 \mathrm{~nm})$ on the eggs and larvae of Atlantic cod (Gadus morhua). Canadian Journal of Fisheries and Aquatic Sciences. 56: 1058-1067.

Belnap, J.; Phillips, S.; Flint, S.; Money, J.; Caldwell, M.

2007 Global change and biological soil crusts: effects of ultraviolet augmentation under altered precipitation regimes and nitrogen additions. Global Change Biology. 14 (3): 670-686.

Björn, L.

1999 Ultraviolet-B radiation, the ozone layer and ozone depletion. In: Rozema, J. (Ed.). Stratospheric ozone depletion. The effects of enhanced UV-B radiation on terrestrial ecosystems. Brackhuys Publishers Leiden. The Netherlands. pp. 21-37.

Cabrera, S.; Bozzo, S.; Fuenzallida, H.

1995 Variations in UV radiation in Chile. Journal of Photochemistry and Photobiology. 28 (2): 137-142.

Caputo, C.; Rutitzky, M.; Ballaré, C.L.

2006 Solar ultraviolet-B radiation alters the attractiveness of Arabidopsis plants to diamondback moths (Plutella xylostella $\mathrm{L}$.): impacts on oviposition and involvement of the jasmonic acid pathway. Oecología 149 (1): 81-90.

Carrasco-Ríos, L.

2009 Efecto de la radiación ultraviolet-B en plantas. Idesia 27 (3): 59-76.

Cockell, Ch.; Mckay, Ch.; Warren-Rhodes, K.; Horneck, G. 2008 Ultraviolet radiation-induced limitation to epilithic microbial growth in arid deserts Dosimetric experiments in the hyperarid core of the Atacama Desert. Journal of Photochemistry and Photobiology B: Biology. 90: 79-87.

Cywinska, A.; Crump, D.; Lean, D.

2000 Influence of UV radiation on four freshwater invertebrates. Photochemistry and Photobiology. 72 (5): 652-659.

Day, T.A.; Neale, P.J.

2002 Effects of UV-B radiation on terrestrial and aquatic primary producers. Annual Review of Ecology and Systematics. 33: 371-396.
Diffey, B.L.

1991 Solar ultraviolet radiation effects on biological systems: Review. Physics in Medicine and Biology. 36(3): 299-328.

Faruki, S.; Das, D.; Khan, A.; Khatun, M.

2007 Effects of ultraviolet ( $254 \mathrm{~nm}$ ) irradiation on egg hatching and adult emergence of the flour beetles, Tribolium castaneum, T. confusum and the almond moth, Cadra cautella. Journal of Insect Science. 7 (36): 1-6.

Faruki, S.; Das, D.; Khatun, S.

2005 Effects of UV- radiation on the larvae of the lesser mealworm, Alphitobius diaperinus (Panzer) (Coleoptera: Tenebrionidae) and their progeny. Journal of Biological Sciences. 5 (4): 444-448.

Faruki, S.; Kundu, P.

2005 Sensitivity of the silkworm, Bombix mori L. (Lepidoptera: Bombycidae) larvae to UV-irradiation. Invertebrate Survival Journal. 2 (1): 75-81.

Frederick, J.E.; Snell, H.E.; Heywood, E.K.

1989 Solar ultraviolet radiation at the Earth's surface. Photochemestry and Photobiology. 50: 443-450.

Foggo, A.; Higgins, S.; Wargent, J.J.; Coleman, R.A.

2007 Tri-trophic consequences of UV-B exposure: plants, herbivores and parasitoids. Oecología. 154 (3): 505-512.

Gliessman, S.

2002 Agroecología. Procesos ecológicos en agricultura sostenible. CATIE. Centro Agronómico Tropical de Investigación y Enseñanza. Costa Rica. 358 p.

González, C.

2007 Evaluación del efecto de la radiación UV-B en la etapa larval de las abejas obreras Apis mellifera L. (Hymenoptera: Apidae) en condiciones naturales, en las ciudades de Valdivia y Concepción. Tesis Licenciado en Ciencias Biológicas. Valdivia, Chile. Universidad Austral de Chile, Facultad de Ciencias, Escuela de Ciencias. 86 p.

Hopkins, W.

1999 Introduction to plant physiology. $2^{\text {a }}$ ed. Wiley, U.S.A. $512 \mathrm{p}$.

Hurtubise, R.; Havel, J.; Little, E.

1998 The effects of ultraviolet-B radiation freshwater invertebrates: experiments with a solar simulator. Limnology and Oceanography. 43 (6): 1082-1088.

Jäckle, H.; Kalthoff, K.

1977 Photoreactivation of RNA in UV-irradiated insect eggs (Smittia sp., Chironomidae, Diptera) I. Photosensitized production and light-dependent disappearance of pyrimidine dimmers. Photochemistry and Photobiology. 27 (3): 309-315.

Johansson, J.; Nyström, P.

2004 Effects of ambient UV-radiation on the behaviour of mayfly larvae of the genus Deleatidium from trout bearing and fishless streams in New Zeland. Archiv für Hydrobiologie. 161 (3): 403-415.

Kalthoff, K.; Urban, K.; Jäckle, H.

1971 Photoreactivation of RNA in uv-irradiated insect eggs (Smittia sp., Chironomidae, Diptera) II. Evidence for heterogeneous light-dependent repair activities. Photochemistry and Photobiology. 27 (3): 317-322. 
Lovengreen, C.; Álvarez, J.; Fuenzalida, H.; Aritio, M. 2002 Radiación ultravioleta productora de eritema en Valdivia. Comparación entre inferencias satelitales, modelo de transferencia radiactiva y mediciones desde Tierra. Revista Médica de Chile. 130: 17-25.

Machado, D.

1986 Efeitos da radiaçao ultravioleta em Apis mellifera (Hymenoptera, Apidae). Monografía. Ribeiráo Preto, Sao Paulo, Brasil. Universidade Sáo Paulo, Faculdade de Filosofia, Ciéncias e Letras de Ribeiráo Preto. 49 p.

Machado, D.

1991 Efeitos da radiaçao ultravioleta em ovos de abelhas africanizadas. Dissertaçáo Mestrado. Ribeiráo Preto, Sao Paulo, Brasil. Universidade Sáo Paulo, Faculdade de Filosofia, Ciéncias e Letras de Ribeiráo Preto. 200 p.

Martin, S.; Cháline, N.; Oldroyd, B.; Jones, G.; Ratnieks, F. 2004 Egg marking pheromones of anarchistic worker honeybees (Apis mellifera). Behavioral Ecology. 15 (5): 839-844.

Martin, S.; Châline, N.; Ratnieks, F.; Jones, G.

2005 Searching for the egg-marking signal in honeybees. Ecology \& Evolutionary Biology. 2: 1-9.

Mazza, C.; Zavala, J.; Scopel, A.; Ballaré, C.

1999 Perception of solar UVB radiation by phytophagous insects: Behavioral responses and ecosystem implications. Agricultural Plant Physiology and Ecology Research. 96 (3): 980-985.

Mazza, C.A.; Izaguirre, M.M.; Zavala, J.; Scopel, A.L.; Ballaré, C.L.

2002 Insect perception of ambient ultraviolet-B radiation. Ecology Letter. 5 (6): 722-726.

Mc Kenzie, R.; Björn, L.; Bais, A.; Iiyasd, M.

2003 Changes in biologically active ultraviolet radiation reaching the Earth's surface. Photochemical and Photobiological Science. 2: 5-15.

Negishi, T.; Nagaoka, C.; Hayatsu, H.; Suzuki, K.; Hara, T.; Kubota, M.; Watanabe, M.; Hieda, K.

2001 Somatic-cell mutation induced by UVA and monochromatic UV radiation in repair-proficient and -deficient Drosophila melanogaster. Photochemistry and Photobiology. 73 (5): 493-498.
Perondini, A.

1984 Photoreactivation of UV-induced cytoplasmatic lesions in eggs of Sciara ocellaris. International Journal of Radiation Biology. 45 (3): 257-260.

Piacentini, R.; Cede, A.; Bárcena, H.

2003 Extreme solar total and UV irradiances due to cloud effect measured near the summer solstice at the high-altitude desertic plateau Puna of Atacama (Argentina). Journal of Atmospheric and Solar-Terrestrial Physics 65: 727-731.

Posso, F.

1999 El agujero en la capa de ozono: origen, evolución y estado actual. Geoenseñanza. 4 (1): 157-189.

Quek, P.H.; Hu, J.Y.; Chu, X.N.; Feng, Y.Y.; Tan, X.L.

2006 Photoreactivation of Escherichia coli following medium-pressure ultraviolet disinfection and its control using chloramination. Water Science and Technology. 53 (6): 123-129.

Rivas, M.; Rojas, E.; Cortés, J.; Santander, E.

2002 Efecto de la altura en la radiación solar ultravioleta en Arica Norte de Chile. Revista Facultad de Ingeniería, U.T.A. 10: 59-62.

Rivas, M.; Rojas, E.; Madronich, S.

2008 Aumento del índice solar ultravioleta con la altura. Revista Chilena de Ingeniería. 16 (2): 383-388.

Rojas, E.; Rivas, M.

2002 Calculations of incident erythemal solar doses in Arica through transfer radiative models. Revista Mexicana de Física. 48 (3): 122-124.

Ruhland, C.; Fogal, M.; Buyarski, C.; Krna, M.

2007 Solar ultraviolet-B radiation increases phenolic content and ferric reducing antioxidant power in Avena sativa. Molecules. 12: 1220-1232.

Sharma, M.K.; Dwivedi, S.C.

1997 Investigation on the effects of ultraviolet and infrared light on the life cycle of Callosobruchus chinensis Linn. Journal of Advanced Zoology. 18: 27-31.

Skerratt, J.H.; Davidson, A.D.; Nichols, P.D.; Mcmeekin, T.A. 1998 Effect of UV-B on lipid content of three Antarctic marine phytoplankton. Phytochemistry. 49 (4): 999-1007.

Solomon, K.

2008 Effects of ozone depletion and UV-B radiation on humans and the environment. Atmosphere-Ocean. 46 (1): 185-202. 
